\title{
ENSEIGNEMENT DES LANGUES ET PRODUCTIONS ORALES DANS LES PROJETS DE TELECOLLABORATION
}

\author{
MARIO TOMÉ \\ Universidad de León
}

\section{Resumen:}

Las aplicaciones de las nuevas tecnologías en la enseñanza de idiomas se abren a diferentes campos de experimentación en la búsqueda de métodos y herramientas que puedan favorecer una buena adquisición de la lengua extranjera así como el conocimiento de su cultura. Las directrices europeas en la enseñanza de idiomas ponen de relieve la importancia de las prácticas orales y de los intercambios interculturales dentro de los actuales contextos educativos en continua transformación. A pesar de la expansión en Internet de herramientas audiovisuales que refuerzan la comunicación, las estrategias de producción oral están aún poco desarrolladas en clase de lengua extranjera. En el marco de proyectos pedagógicos de telecolaboración el lugar que ocupa la lengua escrita es predominante. El proyecto León - Grenoble trata de corregir esta situación equilibrando la práctica oral y la escrita. La utilización de tareas para la producción oral de los estudiantes de francés lengua extranjera (FLE) es aquí fundamental. Y más determinantes aún son las prácticas de corrección de la pronunciación para los tres agentes que intervienen en este contexto educativo: el docente, los tutores y los estudiantes de FLE.

Palabras clave: Enseñanza de idiomas, francés lengua extranjera, producción oral, nuevas tecnologías, proyectos de telecolaboración.

\begin{abstract}
The application of new technologies in language teaching opens up a range of options in the search for methods and tools favourable to both effective foreign language acquisition and knowledge of its culture. In spite of the expansion on internet of audiovisual tools that enhance communication, oral production strategies are still poorly developed in the language classroom. Within the framework of pedagogical telecollaboration projects, written language is usually predominant. The León-Grenoble project tries to correct these discrepancies by trying to establish a balance between oral and written skills. Task-based oral production is here fundamental for learners of French as a Foreign Language (FLE). And even more crucial are the practices to correct pronunciation for the three agents involved in the educational context: teachers, tutors and students of FLE.
\end{abstract}

Key words: Languages teaching, french as foreign language, oral production, new technologies, telecollaboration projects.

\section{Nouvelles technologies en éducation}

$\mathrm{C}$

es dernières années les progrès des nouvelles technologies de l'information et de la communication (NTIC) ont clairement influencé les pratiques d'enseignement et d'apprentissage. Nous avons ainsi différents domaines et notions qui se trouvent en pleine évolution. Les dispositifs de formation utilisant les NTIC se 
diversifient, et on parle ainsi de Formation Ouverte et à Distance (FOAD), de campus virtuels ou numériques. Le terme « dispositif » désigne «un système formel d'apprentissage et un ensemble de moyens matériels et humains, correspondant à une forme de socialisation particulière destinée à faciliter un processus d'apprentissage» (Blandin et al., 2002). En relation avec la notion de «campus virtuel » des auteurs comme Charlier, Deschryver et Peraya (2005) signalent:

Grâce aux dispositifs technologiques actuels - campus numériques, environnements virtuels de travail, etc. - la médiatisation peut porter aujourd'hui sur des objets plus complexes qu'autrefois comme des dispositifs de formation complets, incluant toutes les fonctions pédagogiques et non pédagogiques d'un cours, d'un programme de formation, d'une faculté ou encore d'une université.

Finalement les notions d'E-learning, apprentissage en ligne, E-formation et apprentissage virtuel sont équivalentes en évoquant un mode d'apprentissage basé sur l'utilisation des nouvelles technologies, qui permet l'accès à des formations en ligne, interactives et parfois personnalisées, diffusées par l'intermédiaire d'internet, d'un intranet ou autre média électronique, tout en rendant le processus d'apprentissage indépendant de l'heure et de l'endroit.

L'expansion de l'E-formation et les applications pédagogiques des NTIC se propagent aujourd'hui dans les universités. Nous remarquons l'intégration dans les salles de classe des dimensions et outils suivants: réseaux internet ou intranet, webconférence, plateformes d'enseignement ou de travail collaboratif, weblogs, salles multimédia, projecteurs vidéo, wireless technologies, projets de collaboration interuniversitaire et échanges en ligne entre étudiants, forums et listes de discussion, etc.

La connectivité représente en fait la condition essentielle d'une utilisation efficace des technologies en classe. C'est la capacité des enseignants et des étudiants d'aborder les contenus pédagogiques de façon nouvelle, en utilisant les technologies pour améliorer les processus d'apprentissage. Du didacticiel plus traditionnel à la plateforme de formation, en passant par les sites web dédiés aux cours électroniques ou aux blogs, les nouveaux outils technologiques permettent ainsi de transformer l'environnement traditionnel de la classe, en espace d'apprentissage davantage stimulant et productif.

\section{Nouveaux paradigmes pour l'éducation}

Le rapport de l'UNESCO: L'éducation: un trésor est caché dedans met en relief les quatre piliers de l'éducation:

Pour répondre à l'ensemble de ses missions, l'éducation doit s'organiser autour de quatre apprentissages fondamentaux qui, tout au long de la vie, seront en quelque sorte pour chaque individu les piliers de la connaissance: apprendre à connaître, c'est-à-dire acquérir les instruments de la compréhension; apprendre à faire, pour pouvoir agir sur son environnement; apprendre à vivre ensemble, afin de participer et de coopérer avec les autres à toutes les activités humaines; enfin, apprendre à être, cheminement essentiel qui participe des trois précédents (Delors, J. et autres,1999)

Des auteurs comme Johnson (1992) ou Lundvall (2002) soulignent les notions d'apprendre à faire (learning-by-doing), apprendre à interagir (learning-byinteracting), apprendre à chercher (learning-by-searching) ou apprendre à partager (learning-by-sharing).

Les nouvelles technologies et internet transforment les modes de communication et d'apprentissage, en permettant aux groupes humains de mettre en commun leurs 
imaginations et leurs savoirs. P. Lévy (1997) évoque le projet d'une «intelligence collective » en la définissant comme «une intelligence partout distribuée, sans cesse valorisée, coordonnée en temps réel, qui aboutit à une mobilisation effective des compétences». De Rosnay et Revelli (2006) parlent des "médias des masses", utilisant des techniques numériques de création collaborative, de connexion et d'échange qui s'opposent et dépassent les mass média connus (télévision, radio, éditions, publicité); parmi ces médias des masses, nous pouvons citer aujourd'hui les weblogs, les vlogs (weblogs vidéo), les wikis, le $\mathrm{P} 2 \mathrm{P}$ (peer to peer ou échange de fichiers) et le podcasting (diffusion de fichiers sonores). Enfin, les mutations de l'internet donnent naissance à des notions comme le Web 2.0 ou le E-learning 2.0. S. Downes (2005) signale en relation avec celles-ci:

Educators began to notice something different happening when they began to use tools like wikis and blogs in the classroom a couple of years ago. All of a sudden, instead of discussing pre-assigned topics with their classmates, students found themselves discussing a wide range of topics with peers worldwide. In a very short time, blogs were used for a wide variety of purposes in education, an educational bloggers' network formed and by this year thousands of teachers were encouraging their students to blog. (...) It's not just blogging. Educators have also taken an interest in podcasting. Some have started broadcasting, such as at McMaster, where engineering professors now host an online show.

\section{Apprentissage des langues et NTIC}

Les applications des nouvelles technologies à l'enseignement / apprentissage des langues entrent dans le domaine connu sous le nom d'Apprentissage des langues assisté par ordinateur (ALAO) traduction de l'anglais Computer-Assisted Language Learning (CALL). Une définition proposée par les plus importantes associations internationales en ALAO est la suivante: The field of CALL is inherently multidisciplinary.

It applies from the fields of second language acquisition, sociology, linguistics, psychology, cognitive science, cultural studies and natural language processing to second language pedagogy, and it melds these disciplines with technology-related fields, artificial intelligence and media/ communication studies. (CALICO, EUROCALL et IAALT, 1999).

Dans le contexte francophone l'ALAO est équivalent de l'acronyme ALSIC: Apprentissage des Langues et Systèmes d'information et de Communication. On trouve plusieurs orientations dans ce vaste champ de recherche, ainsi les aspects épistémologiques (Chapelle, Warschauer), le développement des dispositifs en ALAO (Mangenot, Poussard, Rézeau), l'exploitation pédagogique des outils multimédias en classe de langue (Chanier et Pothier), l'analyse des situations d'apprentissage en interaction. Cette dernière concerne le domaine des Apprentissages Collectifs Assistés par Ordinateur (ACAO) traduction de l'anglais Computer Supported Collaborative Learning (CSCL) où l'on trouve des chercheurs en cognisciences, en informatique, en sciences de l'information et de la communication et en sciences de l'éducation (Derycke, Koschmann, Lehtinen, Lund, Salomon).

- Comme signale K. Zourou (2005), ces recherches basées sur les aspects linguistiques et cognitifs des interactions s'ouvrent à des nouvelles perspectives, ainsi une attention particulière est accordée aux spécificités interculturelles (Belz, Thorne) socioaffectives (Lamy, Dejean et Mangenot) ou médiatisées de ces échanges (Thorne, Springer et Aimard). D'un autre côté, la médiatisation de la communication via les réseaux peut être envisagée sous l'angle didactique (comportant des réflexions 
interculturelles) ou sous l'angle de la production discursive qui est le domaine de la Communication médiatisée par ordinateur (CMO).

\section{Les directives européennes pour l'apprentissage et l'enseignement des langues}

Le Cadre européen commun de référence pour les langues: Apprendre, Enseigner, Évaluer (CECRL) publié par le Conseil de l'Europe en 2001, met en relief le but de communiquer pour les apprenants d'une langue, énumère les connaissances et les habiletés nécessaires à un comportement langagier efficace et définit les niveaux de compétence qui permettent de mesurer le progrès de l'apprenant à chaque étape de l'apprentissage.

Ces six niveaux de compétence - utilisateur élémentaire (A1, A2), utilisateur indépendant $(\mathrm{B} 1, \mathrm{~B} 2)$ et utilisateur expérimenté $(\mathrm{C} 1, \mathrm{C} 2)$ - correspondent à la division classique : niveau de base ou débutant, niveau intermédiaire et niveau avancé. Chaque niveau est défini par des compétences de communication à atteindre: écouter, parler (interaction orale, production orale en continu), lire, écrire. Pour chaque compétence, on retrouve un certain nombre de situations de communication ou d'activités; pour chaque situation/activité, on a des descripteurs de compétence qui précisent la tâche à accomplir, la nature ou la difficulté des documents ou informations transmises ou à transmettre.

Parmi les activités de communication langagière on distingue:

- Activités de production : Production orale (Parler) et Production écrite (Écrire).

- Activités de réception: Écoute ou compréhension de l'oral, Lecture ou compréhension de l'écrit et Réception audiovisuelle.

- Activités d'interaction et stratégies: Interaction orale et Interaction écrite.

- Activités de médiation et stratégies: Médiation orale et Médiation écrite.

On peut aussi voir en ligne dans les annexes 1 à 4 (Académie de Toulouse) l'échelle globale et les différents tableaux en relation avec les activités langagières: Ecouter (compréhension auditive), Parler (expression orale), Activités de production orale en continu, Exemples d'activités interactives, Lire (compréhension de l'écrit) et Ecrire (expression écrite).

Pour sa part la Commission européenne signale dans Les grands principes pédagogiques sur lesquels se fonde l'enseignement des langues aux très jeunes apprenants les principes suivants:

- La compréhension doit primer sur la production

- Une motivation positive à l'égard de l'apprentissage

- La formation de l'oreille

- La formation de la prononciation

- L'extension et la formation de la relation entre phonétique et graphèmes dans la langue étrangère.

Et dans l'exposé général des concepts didactiques on remarque la profession d'enseignant, l'utilisation de matériels authentiques, l'apprentissage axé sur les tâches et l'exploitation des technologies de l'information et de la communication.

Finalement el Ministerio de Educación y Ciencia en Espagne suit les directives européennes dans les Reales Decretos de enseñanzas mínimas de la Educación Primaria y de la Educación Secundaria Obligatoria (2006). Dans le document en ligne MECdecretos - Síntesis (RedIRIS) nous avons sélectionné les passages qui font référence aux principaux objectifs, compétences et contenus dans l'apprentissage d'une langue étrangère, ainsi qu'à l'importance des nouvelles technologies de l'information et de la communication dans le panorama éducatif actuel. 


\section{Les tâches pédagogiques avec les NTIC}

Le Cadre européen commun de référence pour les langues se situe dans un perspective actionnelle: parler une langue, c'est agir sur le réel et non reproduire un modèle et l'apprentissage s'effectue à travers la réalisation de tâches langagières les plus authentiques possibles, avec une finalité de communication explicite:

La communication fait partie intégrante des tâches dans lesquelles les participants s'engagent en interaction, réception, production, compréhension ou médiation ou une combinaison de deux ou plus de ces activités comme, par exemple, l'interaction avec un service public et la réponse à un formulaire ou la lecture $\mathrm{d}^{\prime} \mathrm{un}$ rapport suivie d'une discussion avec des collègues pour parvenir à une décision sur un projet, ou le respect d'un mode d'emploi pour réaliser un assemblage et, dans le cas où il y a un observateur ou un assistant, le commentaire ou la demande d'aide sur la procédure, ou encore la préparation (à l'écrit) d'une conférence et la conférence, ou la traduction officieuse pour un visiteur, etc.

Dans un apprentissage axé sur les tâches les technologies de l'information et de la communication ont un rôle déterminant. Comme une autre directive le signalait:

Si l'apprentissage assisté par ordinateur est mis à disposition de manière convenable, une vaste gamme d'informations, d'interaction et de feedback sera possible. Ce résultat démontre peutêtre un nouveau principe pédagogique, à savoir le fait que l'apprentissage assisté par ordinateur mènera à une augmentation en matière d'information, d'interaction et de feedback (Les grands principes pédagogiques sur lesquels se fonde l'enseignement des langues aux très jeunes apprenants, 2006).

Des chercheurs en didactique des langues ont déjà mis en relief l'importance d'une pédagogie fondée sur les tâches pour l'intégration des NTIC en classe et tout particulièrement dans les dispositifs de formation avec les nouvelles technologies. $\mathrm{F}$. Mangenot (2003) remarque que: «La tâche ou le scénario pédagogique incluent une ou des activités faisant sens pour les apprenants, s'appuient sur des ressources et prennent en compte le dispositif spatio-temporel et humain, à la fois en termes de communication et d'accompagnement pédagogique». D. Oliver et J. Herrington (2001) soulignent «que les tâches d'apprentissage constituent l'élément charnière dans le processus de conception de dispositifs constructivistes d'apprentissage en ligne».

Dans ces contextes pédagogiques on a proposé une typologie des tâches (Henri et Lundgren-Cayrol 1997, Mangenot, 2003): la fouille collective, l'analyse critique, le débat, la prise de décision, la résolution de problèmes, la conception de ressources pédagogiques, l'étude de cas. Nous devons ajouter aussi la notion de «projet pédagogique » défini par Mangenot (2005) comme «un ensemble de tâches amenant des apprenants distants à communiquer entre eux. Généralement les projets pédagogiques se font entre classes ou groupes d'étudiants de pays différents afin de favoriser les échanges linguistiques et culturels.»

Dans nos derniers travaux (Tomé, 2006, 2007) nous avons analysé le rôle des tâches, des scénarios et des projets pédagogiques avec accompagnement dans un dispositif hybride type campus virtuel et nous avons expérimenté l'intégration des tâches dans les CMS ou systèmes de gestion de contenu (plateformes et weblogs) pour l'enseignement du français langue étrangère (FLE); nous avons ainsi abordé les modalités suivantes: présentation des étudiants ou de leur ville / région dictées audio; cyberallyes culturels; recherche, sélection et enregistrement des séquences audio disponibles sur le web, 
enregistrement audio des étudiants individuellement ou en groupe à partir d'une tâche pédagogique, lecture d'un texte ou un exercice d'écoute et répétition, création de séquences audio par les étudiants.

\section{Les projets pédagogiques et dispositifs de formation pour le FLE avec les NTIC}

Dans le domaine de la communication médiatisée par ordinateur (CMO) plusieurs recherches ont porté sur les usages communicatifs médiatisés en réseau et la didactique des langues, ainsi que sur la dimension interculturelle des interactions médiatisées entre apprenants. S'il est vrai qu'on peut observer différentes orientations selon que les projets se centrent sur la production langagière, la conscience interculturelle ou les interactions langagières, en tout cas dans l'apprentissage d'une langue étrangère on peut difficilement séparer ces trois composantes essentielles: la langue, la communication et la culture. Nous aborderons ici trois projets interactifs sur internet entre groupes d'apprenants éloignés géographiquement: Cultura, Le français en (première) ligne et le Projet León - Grenoble.

Le projet Cultura a été créé en 1997 par G. Furstenberg, S. Waryn et S. Levet dans le département de Foreign Languages and Literatures du Massachusetts Institute of Technology (MIT) et il se définit comme « une approche comparative interculturelle qui permet à des étudiants français et américains d'élaborer progressivement et collaborativement leur connaissance et leur compréhension des valeurs, attitudes et croyances inhérentes à l'autre culture, en un processus dynamique et interactif de construction réciproque $\gg$. Ce dispositif met en relation deux groupes d'étudiants dans des contextes relativement semblables (deux lycées, deux universités, etc.) qui travaillent ensemble sur les mêmes matériaux (questionnaires) ou échangent sur des forums, généralement dans le cadre de la compréhension et la production écrites.

Le projet Le français en (première) ligne mis en place en 2002 par C.Develotte et F.Mangenot est le résultat dans l'actualité du partenariat suivant: Université de Sydney, Ecole normale supérieure Lettres et sciences humaines (ENS LSH) de Lyon, Université de Franche-Comté, Université Monash de Melbourne, Université Stendha Grenoble 3, Université de Léon en Espagne, Northern Virginia Community College aux Etats-Unis, Université Lyon 2, Université Sophia de Tokyo (Japon) et the University of California, Berkeley (Etats-Unis). «L'idée fondatrice du projet consiste à faire communiquer via Internet des étudiants en master de FLE en France (désormais « étudiants de FLE ») avec des étudiants distants fréquentant des cours de français à l'université, en général à un niveau licence, le français étant pour eux une matière parmi d'autres (désormais « apprenants »). A la différence de la plupart des projets de télécollaboration, les deux publics en contact n'ont donc pas le même statut, ni les mêmes objectifs. Pour les étudiants de FLE, le but est d'une part d'avoir une occasion de pratiquer l'enseignement parallèlement aux cours plus théoriques qu'ils suivent à l'université, d'autre part de se former à l'utilisation des TICE. Pour les apprenants étrangers, il s'agit de leur permettre un contact avec des natifs (ou quasi natifs) de la langue qu'ils apprennent ; ce contact a pour but de leur faire pratiquer la langue dans une situation plus authentique que celle de la classe, en leur fournissant, par le truchement des étudiants de FLE, un accès aux réalités francophones d'aujourd'hui.» (Develotte et Mangenot, 2007). Dans ce dispositif les échanges en ligne se déroulent pour la plupart en mode asynchrone dans le cadre d'une plateforme d'enseignement où l'on a développé la compréhension et la production écrites et exceptionnellement la compréhension orale. C'est à partir de 2005 que les productions orales commencent à 
faire partie des tâches pédagogiques, et plus récemment on a réalisé quelques expériences vidéo, mais dans l'ensemble ce projet a privilégié la modalité écrite.

Le projet León - Grenoble créé en 2005 dans le cadre du projet précédent est le résultat du partenariat entre l'Université Stendhal - Grenoble 3 (F. Mangenot, laboratoire Lidilem) et l'Universidad de León en Espagne (M. Tomé, Campus Virtuel FLE - Projet Flenet). Cette recherche-action consiste d'une part à faire réaliser par des étudiants en master de français langue étrangère (Université de Grenoble) des tâches multimédias pour des étudiants distants (Université de León), d'autre part de susciter des échanges en ligne entre les deux publics autour de ces tâches. On remarque comme objectifs principaux du projet: favoriser une communication authentique et des échanges linguistiques et culturels dans le contexte d'apprentissage du FLE, développer la compréhension et la production orales chez les étudiants espagnols et réaliser des expériences pédagogiques avec les NTIC en classe de français (création de tâches, utilisation de la plateforme Moodle, outils informatiques audio-visuels, dispositif de formation à distance, tutorat). A différence des projets antérieurs ce dispositif veut équilibrer les productions écrites et les productions orales. Pour renforcer ce décalage on a adopté une approche fondée sur des tâches de compréhension et production orales, ainsi que sur un bon accompagnement dans la correction de la prononciation par les tuteurs français. Dans l'actualité on poursuit ces expériences en introduisant la vidéo soit pour des émissions en direct soit pour l'enregistrement de séquences autour des tâches réalisées, grâce à la communauté de live broadcasting vidéo UStream TV.

\section{Les outils informatiques}

Moodle est une plate-forme d'apprentissage en ligne (e-learning) sous licence open source servant à créer des communautés d'apprenants autour de contenus et d'activités pédagogiques. Ce CMS ou système de gestion de contenu dispose de remarquables fonctions communicatives pour créer un environnement d'apprentissage en ligne et permettre des interactions entre des pédagogues, des apprenants et des ressources pédagogiques. Ce système de e-formation peut aussi être défini comme un dispositif de formation ouverte et à distance (FOAD) ou bien comme un environnement d'apprentissage médiatisé (Voir Moodle - Wikipédia: http://fr.wikipedia.org/wiki/Moodle). Pour le développement du Projet León-Grenoble nous nous servons fondamentalement de la fonction "Forum", puisque ces espaces rendent possible une communication souple grâce à l'envoi de messages écrits (tâches, informations, questions) ou des pièces attachées (documents, images, fichiers audio et vidéo). Les différentes années du projet constituent des cours à l'intérieur de Moodle Dpto.Filología Moderna qui est logé dans les serveurs de l'Université de Leon à l'adresse web: http://www3.unileon.es/dp/dfm/moodle/. L'image suivante montre la page générale du projet León-Grenoble 2007-2008: 


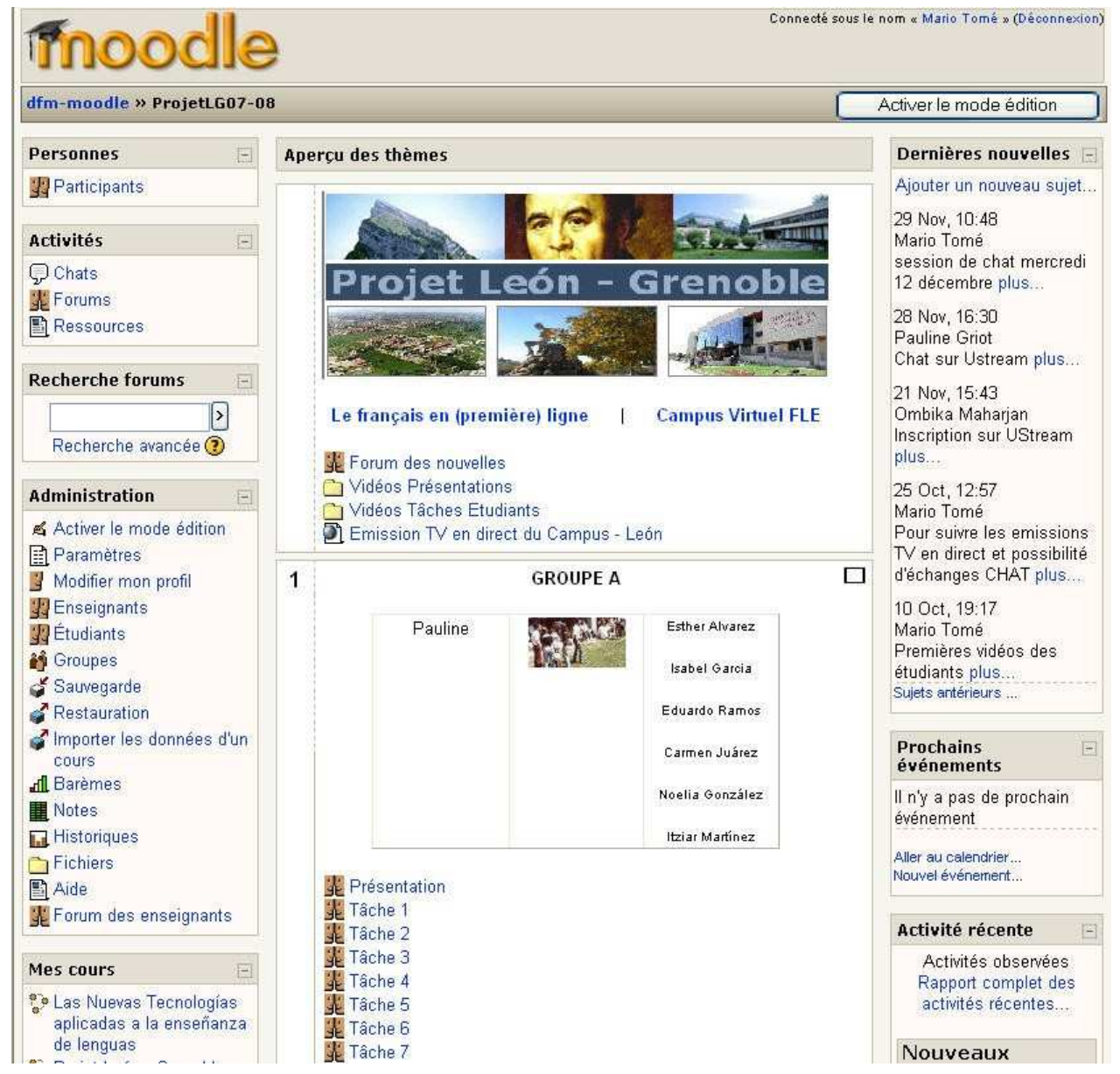

tepVoice Recorder est un petit programme gratuit et d'application qu'on installe dans les système Windows de notre ordinateur. Il permet d'enregistrer simplement et directement toutes les sources de sons au format mp3. A l'aide d'un microphone les étudiants créent facilement des fichiers audio pour la réalisation de leur tâches pédagogiques. Les tuteurs ont utilisé souvent Audacity, un logiciel gratuit plus complexe qui constitue un vrai studio d'édition audio. Les étudiants de l'Université de Léon ont utilisé occasionnellement des magnétophones ou enregistreurs web que l'on trouve sur des hébergeurs de blogs audio ou podcasting, comme par exemple: Podomatic, Odeo ou Jamglue.

UStream TV (site web: http://www.ustream.tv) est une communauté de live broadcasting en vidéo (émission en direct) qui rend possible la retransmission de séquences vidéo à partir d'une simple connexion de webcam, ce qui permet de faire des webconférences ou des émissions de télévision. Nous nous servons de cet outil pour mettre en contact plus réel les étudiants de l'Université de León et les tuteurs de l'Université de Grenoble ; ainsi que pour enregistrer des extraits vidéo des activités pédagogiques que les étudiants réalisent en salle informatique. La préparation et l'enregistrement de ces séquences vidéo favorisent la création de situations de communication authentiques dans lesquelles les étudiants actualisent leurs compétences langagières, travaillent en collaboration et réfléchissent sur l'acquisition d'une langue étrangère. Sur le canal Campus de UStream $T V$, disponibles en ligne : http://www.ustream.tv/campus, nous pouvons consulter des tâches vidéo comme les présentations des étudiants, EduardoFatima Tâche orale, Noémie accompagnement Etudiants, Enrique Mode Emploi Campus, CoralAsierPatricia Tâche orale, Aurélien 
accompagnément, Isabel Tâche enregistrement ou plusieurs Tâches Orales de correction entre étudiants.

\section{Les productions orales dans les projets de télécollaboration}

Comme nous l'avons signalé antérieurement, la plupart des projets de télécollaboration entre étudiants éloignés géographiquement n'ont pas pour objectif ou négligent partiellement ou complètement les productions orales des apprenants. Il y a bien sûr des expériences de production orale dans des dispositifs conçus pour parlerécrire en ligne en temps réel comme le système Lyceum, in Open University (Vetter 2004, Lamy 2004, 2006, Reffay et Betbeder 2006). Nous disposons de recherches remarquables sur l'utilisation de la vidéoconférence pour l'enseignement des langues (O'Dowd, 2006, Develotte, Guichon et Kern, 2007). Et spécialement le travail d'A.Marcelli, D.Gaveau, et R. Tokiwa (2005) qui déclarait comme objectif principal favoriser les compétences orales des apprenants par le biais de tâches communicatives, constatant que «l'utilisation de la visioconférence fait appel à une réelle pédagogie de communication » et affirmait que cette utilisation:

nous apparaît comme un véritable moyen de favoriser l'actualisation et le développement des compétences orales en FLE des apprenants, tant du point de vue de la compréhension que de l'expression. En effet, ce dispositif permet de valoriser l'oral en produisant un effet de face-àface aux différents interlocuteurs qui participent de ce fait à une communication multicanal, c'est-à-dire mimo-gestuelle et verbale, sollicitant une écoute active et une attention soutenue de la part des interactants.

Depuis 2005 le projet Le français en (première) ligne et le Projet León - Grenoble sont pionniers dans le développent des stratégies de production orale et, ce qui est encore moins habituel, dans la correction de la prononciation. Nous présentons par la suite quelques exemples de tâches qui ont pour but la production orale, les productions audio et vidéo des étudiants espagnols ainsi que les pratiques audivisuelles de correction de la prononciation.

\section{Les tâches pédagogiques pour la production orale}

Sans prétendre proposer une typologie exhaustive des tâches pédagogiques visant à une production orale nous résumons les suivantes élaborées par les tuteurs (étudiants de Master FLE) de l'Université de Grenoble :

A. Ecoute et enregistrement des mots, phrases ou chanson en relation avec des sons ou des oppositions phonologiques qui impliquent un certain degré de difficulté pour les étudiants espagnols. Exemples:

- Ecoutez le dialogue 2 . Rejouez le dialogue à deux et envoyez-nous l'enregistrement. Attention aux sons [y] et [u] (PLG2005, Groupe B, Tâche 3).

- Participez au casting pour la nouvelle comédie musicale. « Le roi Dagobert» Chantez un couplet de la chanson et devenez la star de demain. Maintenant vous savez comment prononcer les mots de cette chanson.Dicutez entre vous pour que chacun 
chante un couplet différent. Lisez attentivement le couplet de votre choix , et préparer vous pour le chanter. Puis chantez - le et envoyez nous l'enregistrement (PLG2006, Groupes CD, Tâche 5).

- Maintenant, enregistrez-vous pour chanter ce couplet. Attention à la prononciation du son /OE/ ! PLG2007, Tâche 3,Activité 1).

B. Enregistrement d'une séquence audio dans laquelle un étudiant se présente lui-même ou une autre personne; il peut aussi faire une présentation à deux sous forme de dialogue ou interview. Exemples:

- Maintenant, vous savez poser des questions et y répondre! Par deux, présentez-vous sous forme d'interview et enregistrez-vous. Ajoutez des photos !!! (PLG2005, Groupe A, Tâche 1)

- Réalisez un dialogue de présentations avec un camarade et enregistrez-vous selon le modèle suivant : - Salut comment t'appelles tu ? - je m'appelle ... et toi ? - moi, je m'appelle... (PLG2007, Tâche 1 Activité 1).

C. Enregistrement d'une séquence audio dans laquelle l'étudiant a préparé un texte, un dialogue, une description ou répond à un questionnaire. Exemples:

- Décris à l'oral Lucie, Paul et le couple. Tu peux parler de leurs vêtements, de leurs cheveux, de leurs chaussures...(PLG2005, Groupe D, Tâche 4)

- Exercice 3: Par deux, choisissez une situation. Imaginez un dialogue et enregistrezvous. 1. Un jeune garçon rencontre sa voisine qui se promène avec son chien. 2. Un étudiant espagnol rencontre ou fait connaissance avec un étudiant chinois à l'arrêt du tramway. 3. Un présentateur radio fait une interview à une chanteuse célèbre. (PLG2006, Groupe C, Tâche 2)

- Scénario: Vous êtes le serveur du restaurant. Donnez à Cathy et Mathieu le maximum d'indications spatiales pertinentes pour les rassurer et leur donner des repères. Utilisez les annexes 1 et 2. Ecrivez vos indications puis enregistrez-vous ! Exemple pour Cathy : «Mademoiselle, la corbeille à pain est au milieu de la table, à gauche. » (PLG2007, Tâche 5, activité 3) 
Re: Tâche 4 - Activité 3

par Aude Diancoff - Wednesday 28 November 2007, 12:07

Salut Asier et Raquel!!

舅 correction-raquel_et_asier.mp3

Votre dialogue est bien fait !! Bravo à tous les deux !!

- Attention au son [Y] (tu, université, super)

- Raquel tu as fait les liaisons, c'est bien !!

- Asier, tu as bien prononcé le son $/ \mathrm{OE} /$, c'est génial I! $\mathrm{F}$ ais attention à la liaison entre suis et en (je suis_en train de...). Ecoute l'enregistrement : on ne peut pas mettre le pronom te devant peux comme en espagnol, te puedo decir = je peux te dire (le pronom té est lié au verbe dire - dire à qui ? à toi.)

Voici votre dialogue corrigé

A: Raquel! Ça fait longtemps!

R: Oui Asier! Ça va?

A : Ça va bien. Que' est-ce que tu as fait depus ?

R: J'ai fini l'université. Et toi?

A: Je suis en train de terminer mon doctorat.

R: C'est super!

A : Est-ce que tu vois manger avec moi? Je te peu dire comment $\mathrm{J}$ ' ai été.

R:C'est bien, Mardi 8 heures?

A : D'accord, á Mardi.

R: Au revoir !

Je vous envoie aussi l'enregistrement de votre dialogue corrigé!!

Bises

Aude

\section{Les productions orales des étudiants de FLE}

A partir des tâches pédagogiques antérieures les étudiants espagnols préparent individuellement ou à deux les enregistrements. Ils se servent généralement du programme StepVoice Recorder, un petit logiciel qui permet d'enregistrer simplement et directement sur l'ordinateur au format mp3. Ensuite ils envoient le fichier son comme une pièce attachée dans le message de réponse ou réalisation de la tâche. On peut consulter en ligne quelques exemples sur la rubrique Audio du Projet León Grenoble http://flenet.rediris.es/projetLG/webProjetLG05.htm\#audios

Tâche Orale Présentation Aida: «Bonjour, je m'appelle Aida. J'ai 18 ans. J'aime le cinéma, la musique et le théâtre». (PLG2005, Groupe C, tâche 1).

Tâche Orale Présentation de l'Université - Dialogue entre Sergio et Lily: Sergio: «Bonjour, nous sommes Lily et Sergio et nous allons parler de notre université. Notre université c'est très grande et les professeurs sont très agréables et bons». Lily: «Elle est un peu loin du centre, c'est pourquoi nous devons aller dans le bus. Nous sommes étudiants de philologie anglaise.» (PLG2005, Groupe A, tâche 2).

Tâche Orale à partir d'un scénario : Vous êtes le serveur du restaurant : Coral: «Mademoiselle, vous êtes dans la salle du restaurant, les toilettes se trouvent à gauche du salon, qui est devant cette salle. Mais vous devez faire attention pour y aller parce que il y a des tables, vous devez faire attention aussi aux portes pour sortir! Monsieur, vous êtes dans la salle du restaurant, le salon se trouve derrière vous. Si vous voulez sortir de cette salle, vous pouvez aller tout droite, où est la sortie.» (PLG2007, Tàche orale 5, activité3)

Nous faisons aussi des expériences avec l'enregistrement vidéo des séquences où les étudiants préparent leurs tâches audio ou sont accompagnés par un enseignant ou tuteur. Pour rendre souple et facile cette activité nous nous servons de la fonction enregistrement avec une webcam dans le cadre des émissions en direct d'UStream TV. Le but de ces séquences vidéo est de renforcer la communication avec les tuteurs de l'Université de Grenoble, qui peuvent suivre en direct la réalisation des tâches par les 
étudiants de l'Université de León. Cette communauté de live broadcasting permet en même temps grâce à un espace chat (salon de bavardage) de communiquer par écrit. Nous investissons en ce moment dans des stratégies de production orale organisant des rencontres en direct dans lesquelles les tuteurs à distance posent des questions écrites dans le chat et les étudiants répondent à l'oral à l'intérieur d'une émission UStream TV. Pour avoir une idée de ce type d'activités on peut consulter en ligne la rubrique Vidéo du Projet León - Grenoble http://flenet.rediris.es/projetLG/webProjetLG05.htm\#videos ainsi que le canal Campus de UStream TV: http://www.ustream.tv/campus. Nous transcrivons par la suite quelques exemples.

Tâche Audiovisuelle Dialogue présentation Paula et Enrique: Enrique: «Salut. Comment tu t'appelles?» Paula: «Je m'appelle Paula et toi?» E : «Je m'appelle Enrique.» $\mathrm{P}:$ «Et quel âge astu ?» $\mathrm{E}$ : «Comment? Ah, quel âge.. Euh J'ai dix-huit ans. Et toi ?» $\mathrm{P}:$ «Je suis trente ans.»E : «Et où est-ce que tu habites ?» $\mathrm{P}:$ «J'habite à León. Et toi ?» E : «Moi, j'habite aussi.» (PLG2007, Tâche orale 1).

Tâche Audiovisuelle Dialogue préférences Coral et Asier : Asier : «Quel est ton livre préféré ?» Coral : «Mon livre préféré c'est « La sombra del viento » $\mathrm{A}$ : «Quel est ton sport préfére ?» $\mathrm{C}$ : «J'adore le basket.» A : «Comment s'appelle ton chanteur ou chanteuse favori ?» C: «Mon chanteur favori s'appelle Alejandro Sanz» A : «Quel est ton film préféré ?»C : «Gladiateur» A : «Et comment s'appelle ta meilleure amie ?» C: «Ma meilleure amie s'appelle Elena.» (PLG2007, Tâche orale 2).

Tâche Audiovisuelle Dialogue Patricia et Diego : Patricia : «Bonjour Aude, nous sommes en train de faire l'activité 3 de la tâche 4. Oh!! mais Diego!! je suis Patry!!» Diego: «Ah !! Qu'est ce que tu as fait ces derniers ans?» $\mathrm{P}$ : «J'ai étudié l'anglais et maintenant je suis traducteur dans un journal, ¿et toi?» D: «Je suis ingénieur, et je suis toujours avec ma petite amie de l'université.» P: «ah ! Rigoberta? et elle va bien?» D: «Oui, elle est médecin à l'hôpital de Leon» $\mathrm{P}$ : «Ok, tu veux nous voir demain au café» $\mathrm{D}$ : «Ok, à 8 heures; par exemple». $\mathrm{P}$ : «Ok, à demain?» D: «A demain!» (PLG2007, Tâche orale 4).

Les productions orales des étudiants espagnols correspondent àc leurs niveaux différents de maîtrise de la langue et à leur expérience inégale dans l'acquisition de la prononciation. Le groupe de première année de faculté rassemble des vrais débutants avec une moyenne d'étudiants qui ont déjà étudié le français au collège ou au lycée pendant 2 ou 4 années scolaires. Nous observons les difficultés et interférences articulatoires qui caractérisent les locuteurs hispanophones (Tomé 1994, 1995), c'està-dire, les voyelles ( [y], /OE/ et les nasales), les semi-voyelles ( [j] yod, [4] ) et les consonnes $([\mathrm{v}],[\mathrm{z}],[]],[3],[\mathrm{R}])$, ainsi que différents problèmes en relation avec le rythme et l'intonation.

\section{Les pratiques de correction de la prononciation}

F.Mangenot et K.Zourou (2007), dans le chapitre Corriger en ligne: une activité complexe et chronophage, abordent la problématique de la correction de la prononciation de la part des étudiants-tuteurs de l'Université de Grenoble et remarquent que la question du feed-back par rapport aux productions orales asynchrones constituait une totale nouveauté ce qui a provoqué de nombreux échanges chez les tuteurs. Nous notons les propos suivants comme particulièrement révélateurs: 
La plupart des étudiants disent s'être interrogés sur la question de la correction des erreurs (cf. SR-B1-a, SR-C2-a, SR-C2-b, SR-D1-b, SR-E1). Celle-ci était certes ressentie comme incontournable (ESD-A1-a, b), mais à la fois compliquée à mettre en œuvre et dévoreuse de temps. La complexité de la correction à distance provient à la fois de la moindre interactivité par rapport au présentiel (ESD-E2-a, b) et du fait d'avoir à corriger des enregistrements sonores, activité totalement inédite pour tous les tuteurs (SR-C2-e, ESD-C1-d). Dans les ESD (extraits non fournis ici), les étudiants français disent avoir passé au moins autant de temps, si ce n'est plus, à corriger qu'à concevoir les tâches, ce qui est confirmé dans (SR-D1-a). La dimension chronophage (évoquée dans SR-C2-d et ESD-C1-d) a donc amené certains à s'organiser de manière plus efficace (SR-C2-d, SR-D2-a), en établissant des règles avec les apprenants (SR-D1a), en se répartissant les apprenants ou les tâches à corriger (ESD-E1-a) ou en renonçant à corriger systématiquement (ESD-C1-c). (...) Pour terminer sur la manière dont les tuteurs ont vécu les corrections, on signalera deux extraits de SR dans lesquels les intéressés soulignent de manière très pertinente le lien entre leur travail de conception de tâches et leur travail de correction : SR-C2-a et SR-E2.

Ces auteurs signalent en même temps trois techniques utilisées par les étudiantstuteurs pour effectuer la correction de la prononciation en relation avec les fichiers audio ou séquences sonores réalisées par les étudiants de l'Université de León. Nous les transcrivons par la suite:

Une première technique consiste à utiliser l'écrit pour réagir aux productions, mais cela supposait également l'emploi de l'Alphabet Phonétique International (API); ce type de feedback peut alors contenir des commentaires généraux, des signalements de problèmes à différents niveaux langagiers et des corrections phonétiques plus précises, appuyées sur l'API (voir extraits 12 et 13).

Une seconde technique, la moins coûteuse en temps, consistait à réenregistrer, avec la voix d'un locuteur natif, le message envoyé par les Espagnols: on est alors proche d'une fonctionnalité présente dans de nombreux cédéroms de langues et permettant de comparer sa prononciation à celle d'un natif, sachant qu'un apprenant, du fait du crible phonologique, n'est pas forcément capable de discriminer les phonèmes qui lui posent problème.

La troisième technique demandait un peu plus de maîtrise technologique (cf. SR-C2-c) ... consiste à insérer la voix du tuteur natif à l'intérieur même de l'enregistrement originel, par segments en général brefs, de l'ordre de la phrase ou du mot. Cette technique présente trois avantages : tout d'abord, la charge mémorielle pour l'apprenant qui écoute le message est moindre que dans la technique 2 ; ensuite, le tuteur peut, pour chaque groupe de mots mal prononcé, insister par l'intensité et l'intonation de sa voix, sur telle ou telle erreur de prononciation; enfin, à l'instar des réponses aux messages venant s'insérer dans le texte originel, cette technique crée une certaine impression d'interactivité. (Mangenot et Zourou (2007), Pratiques tutorales correctives via Internet : le cas du français en première ligne).

Dans les exemples suivants nous pouvons nous faire une idée plus claire de ces différentes pratiques de correction de la prononciation. Ainsi pour les corrections qui ont recours à l'écrit nous notons:

Réponse écrite pour la correction de la prononciation : C'est bien dans l'ensemble mais il y a des efforts à faire pour les [s] / [z], [e] / [oe], les [g], les liaisons... Quand ces erreurs "typiques" disparaitront vous aurez fait un grand progrès!! C'est bien aussi que vous vous corrigiez entre vous (autobus!!). Continuez dans ce sens avec régularité et c'est gagné ! (PLG2005, TutriceGA, CorrectionOralUniversite) 
Extrait texte correction prononciation: Bárbara : «Qu'est-ce que tu aimes ou n'aimes pas?» Iván : «'aime sortir en fête (faire la fête) ,aller au cinema, écuter (écouter) de la musique, être avec mes amis et sourtout $([\mathrm{u}])$ voyager([ja]) Je n'aime pas les legumes, me lever tôt, étudier, (les) maths... tu dis «le » au lieu de «les » c'est [e] : les légumes, les maths» Bárbara : «Qu' estce que tu as fait dans les jours fériés qu'on a eu en octobre ?» Iván : «Je suis allé a (dans) un village dans la montagne où j'ai des amis.en plus je suis sorti vendredi soir avec mes amis a (à) León. Tu dis «Et en » avec une liaison. Attention il n'y en a pas ici.» (PLG2006, TutriceGB, InterviewIvan)

Extrait texte correction prononciation: Ta prononciation est bonne. Commencer une nouvelle langue n'est pas facile et c'est normal. On doit habituer notre oreille à de nouveaux sons. Dans les corrigés audio que je t'ai envoyés $1 /$ je te montre comment se prononce " est » [e] 2/ et comment fonctionnent les liaisons: - il est chanteur [ilechãtoeR] pas de liaison devant une consonne - il est acteur [iletaktoer] liaison devant une voyelle Allez courage! A très bientôt (PLG2007, TutriceGA, EduardoT1a3)

Réponse écrite en document MSword accompagné de liens de correction audio sous forme d'images: Bravo pour ton enregistrement et merci de m'avoir présenté Raquel !! Ecoute la correction de ton enregistrement IMAGEhaut-parleur : Fais attention à certaines choses qu'on a déjà vues: - On ne fait pas de liaisons après et Ex : et ses amis [esezami] • N'oublie pas de faire la liaison dans les autres cas Ex : ses meilleurs_amis ou ses_amis [sezami] • Tu as lu "esport" IMAGEhaut-parleur au lieu de "sport" IMAGEhaut-parleur. Ne prononce pas /Es/ mais /s/. (PLG2007, TutriceGC, AsierT2a3)

Extrait correction Audio de la prononciation; dans cette transcription nous notons en souligné les erreurs de l'étudiant et entre parenthèse la correction de la tutrice : Je m'appelle Alexis. Je suis devant un ordinateur. Je me trouve derrière une table. Je suis à côte de Mercedes (Tutrice : Je suis à côté de Mercedes). Je porte un pull bleu, gris et blanc (Tutrice : Je porte un pull bleu, gris et blanc). Je suis châtain. Il y a un livre sur la table. Je porte lunettes (Tutrice : je porte des lunettes). (PLG2006, TutriceGC, audioalexis)

Réponse dans le forum où l'on propose une correction de la prononciation à l'aide d'un document vidéo qui présente la position articulatoire de la bouche pendant l'émission d'un phonème : Bonjour à tous! J'ai écouté vos enregistrements de la phrase "Les secrets les mieux gardés sont ceux qui jamais n'ont été demandés". Ce n'est pas mal dans l'ensemble, mais il faut insister sur le son e (comme dans "secrets"). Pour cela, nous avons "filmé" la phrase. Regardez la vidéo (video_phonetique_3.avi), observez la position de la bouche, puis essayez de faire la même chose (en regardant dans un miroir ou en travaillant par deux). Si vous le voulez, vous pouvez nous envoyer un nouveau fichier son en $\mathrm{mp} 3$ pour voir si votre prononciation est maintenant parfaite!!! (PLG2005, TutriceGA, correction générale de la prononciation des sons é/e).

\section{Corrections collaboratives audiovisuelles entre étudiants}

Nous devons aborder ici d'autres pratiques de la correction de la prononciation qui se produisent entre les étudiants pendant le temps de préparation des tâches ou favorisées par certains contextes pédagogiques comme l'enregistrement de séquences vidéo adressées aux tuteurs de l'Université de Grenoble. C'est ce que nous pourrions définir comme correction phonétique en collaboration. Ainsi nous pouvons parler de corrections entre étudiants spontanées lorsqu'ils préparent leurs tâches orales soit individuelles ou en groupe dans lesquelles ils se posent des questions, répètent des mots ou des phrases à haute voix avant de s'enregistrer et parfois s'autocorrigent. Comme nous n'avons pas d' échantillons de ces situations car elles n'ont pas été enregistrées ni passées à l'écrit, nous avons décidé de les récréer dans un contexte de tâche ou scénario pédagogique. Nous avons proposé aux étudiants de les filmer comme dans une 
émission en direct avec UStream TV. C'est ainsi que nous avons obtenu des séquences audiovisuelles de correction phonétique en collaboration comme celles que nous transcrivons par la suite:

Séquence audiovisuelle avec UStream TV. Tâche orale de correction de la prononciation en forme de dialogue entre deux étudiants à partir de la lecture d'un texte crée dans la cadre d'une tâche proposée par les tuteurs: Esmeralda: Salut Ombika, nous allons préparer les tâches 5 et nous allons enregistrer. Lidia: Salut. Monsieur, la dame de saumon est à droite dans votre assiette. Le riz sauvage est à milieu de votre assiette. E: Attention, comme la vache: Meuh!! Le . L: Le. Les crevettes sont à gauche dans votre assiette. Finalement, le cocktail est devant votre assiette. E: le, le cocktail L: Le cocktail Mademoiselle, le verre d'eau et le verre de vin rouge sont devant votre assiette. $\mathrm{E}$ : le, le verre $\mathrm{L}$ : le verre, le verre de vin rouge sont devant votre assiette (PLG2007, Esmeralda et Lidia, Grupe B: tâche 5, activité 3).

Séquence audiovisuelle avec UStream TV. Tâche orale de correction de la prononciation en forme de dialogue entre deux étudiants à partir de la lecture d'un texte crée dans la cadre d'une tâche proposée par les tuteurs : Esther: Bonjour Pauline, nous allons préparer la tâche 5 activité 3. Monsieur et Mademoiselle, la sortie est au fond du restaurant et votre table est à côte des accès cuisine. $C$ : On dit à côté de. E: à côté de. C: et aussi cuisine comme ZZZ. E: cuisine, cuisine. C: ZZZ E: cuiZZine C: Continuez. E: devant de la sortie. Le bar, les toilettes et le salon C: Attention on dit EUH!! comme la vache française. E: Le bar, les toilettes et le salon sont derrière vous. Les toilettes sont à gauche et le salon est à droite. C: Vous pourrez faire plus d'effort articulatoire dans "salon", O , On, les nasales. E : Et le salon est à droite. (PLG2007, Coral et Esther, Grupe A: tâche 5, activité 3).

Séquence audiovisuelle avec UStream TV. Tâche orale de correction de la prononciation en forme de dialogue entre deux étudiants à partir de la lecture d'un texte crée dans la cadre d'une tâche proposée par les tuteurs: Patricia: Bonjour Aude nous sommes en train de faire la tâche 5. Diego: Pour se dégourdir les oreilles, P: Attention: dégourdir D: dégourdir P: Attention: les oreilles D: les oreilles, rien P: Attention, rien, comme E En, rien D: rien de tel que ce bar restaurant P: restaurant D: où l'on peut apprécier soit un cocktail, soit un repas surprise dans le noir P: attention A An dans D: dans le noir. (PLG2007, Patricia et Diego, Grupe C: tâche 5).

Ces activités pédagogiques se révèlent très efficaces pour favoriser la correction de la prononciation, car les étudiants doivent réfléchir et se poser des questions méthodologiques pour améliorer leur prononciation, appliquer leurs connaissances et compétences dans un contexte réel pour résoudre des problèmes, ainsi que rechercher des outils et des stratégies qui rendent possible une correction effective dans une situation de communication authentique; non seulement parce qu' ils participent à un dialogue, mais aussi parce qu'ils savent qu'ils vont être regardés et écoutés par les tuteurs ou d'autres étudiants de la classe. 


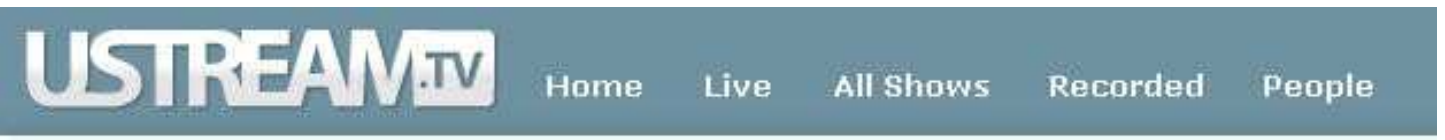

\section{CAMPUS'S RECORDED VIDEOS}

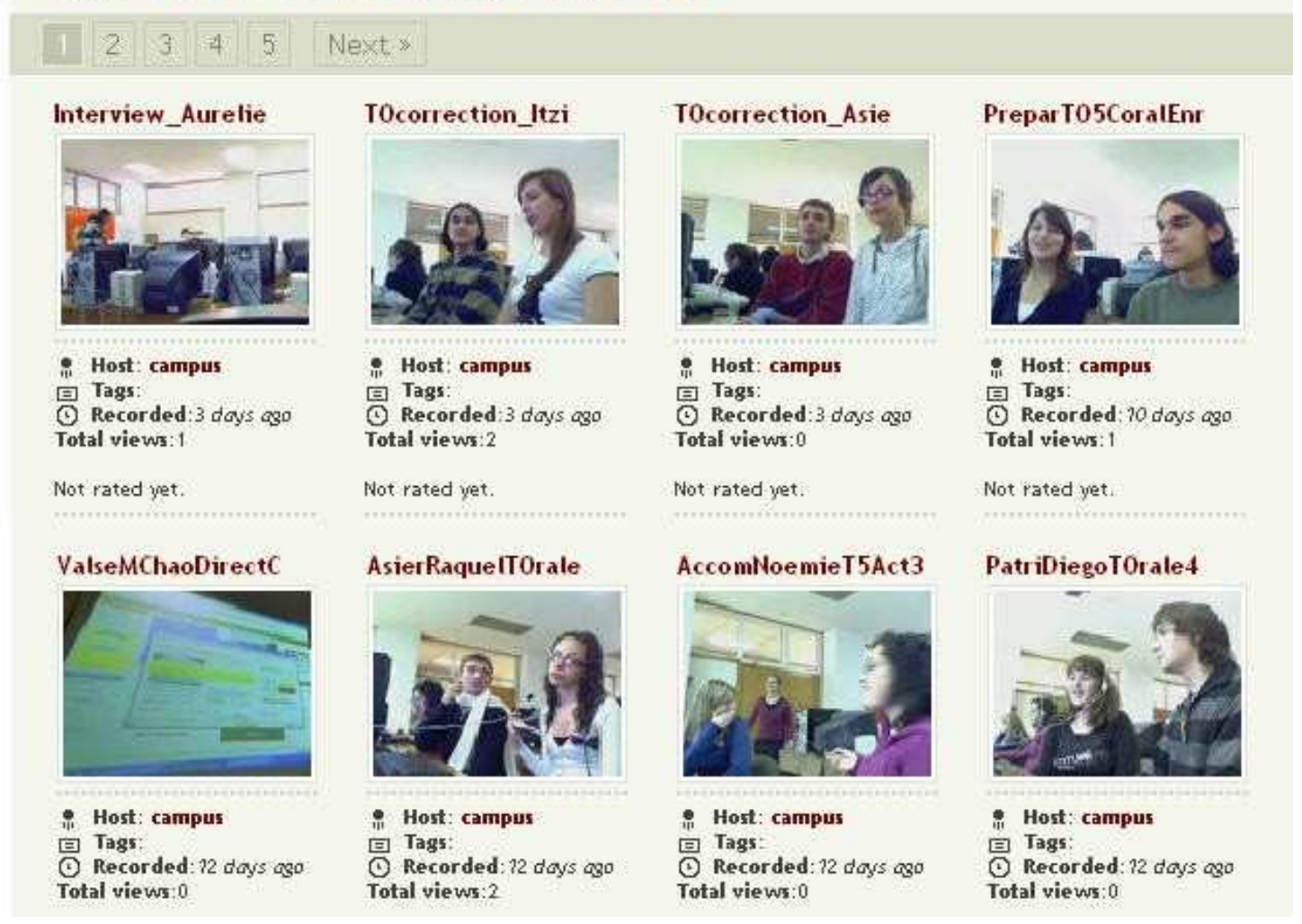

\section{Conclusions}

Malgré l'essor des nouvelles technologies qui transforment notre société et tout particulièrement le monde de l'éducation il y a encore un long chemin à faire pour arriver à leur intégration convenable dans nos universités et nos salles de classe. Les nouveaux outils et dispositifs pour l'enseignement doivent se conjuguer avec les actuels besoins et paradigmes éducatifs comme apprendre à faire, apprendre à interagir, apprendre à chercher ou apprendre à partager. Dans l'enseignement des langues ces outils de communication favorisent des pratiques plus authentiques pour l'acquisition des compétences langagières ainsi que pour les échanges avec d'autres cultures. Les directives européennes pour l'apprentissage des langues nous rappellent toujours l'importance des activités de production orale, d'interaction orale et de médiation orale. Mais la place de l'écrit continue à être prépondérante dans la plupart des ressources éducatives et dans les pratiques pédagogiques qui se servent des NTIC et Internet.

Nous trouvons cette même situation dans le domaine des projets de télécollaboration pour l'enseignement des langues. Grâce aux nouvelles technologies la communication entre étudiants de pays différents est plus facile et l'on dispose de toutes sortes de ressources langagières et culturelles. Mais les productions orales si déterminantes dans l'apprentissage d'une langue étrangère ont un rôle secondaire ou accessoire dans la plupart de ces projets de télécollaboration. C'est pourquoi dans le cadre du projet León - Grenoble nous avons voulu équilibrer et corriger ces décalages en investissant dans 
les stratégies pédagogiques de production orale pour les étudiants espagnols de français langue étrangère.

Comme de nombreux chercheurs en didactique qui ont remarqué l'importance d'une pédagogie fondée sur les tâches, spécialement dans les dispositifs de formation avec les nouvelles technologies, les enseignants des deux universités partenaires (Grenoble et León) ont favorisé la création de tâches pédagogiques comme composante essentielle qui soutient et articule les échanges et la communication entre les tuteurs de l'Université de Grenoble et les étudiants de l'Université de León. Et pour renforcer la pratique des productions orales chez les étudiants espagnols il fallait que les tâches proposées par les tuteurs français favorisent cette pratique. C'est ainsi que ceux-ci ont trouvé différentes modalités de tâches comme l'écoute et enregistrement des séquences sonores en relation avec les phonèmes du français ou l'enregistrement audio de présentations, dialogues ou interviews. A ce niveau le degré d'interactivité et communication a été très efficace et productif, car les étudiants espagnols ont toujours compris les consignes et ils ont mis les moyens pour réaliser les tâches orales, en étant généralement conscients que ce type d'activité était aussi important et complémentaire de la réalisation des tâches écrites.

Comme il était prévisible, les productions orales des étudiants espagnols reproduisent des systèmes d'erreurs et des problèmes articulatoires typiques des locuteurs hispanophones. Mais l'élément décisif dans ces productions orales est qu'elles se situent dans un vrai contexte de communication, car les étudiants espagnols savent qu'ils vont être écoutés par les tuteurs français, qu'ils recevront une réponse d'aide ou de correction et que leur travail a du sens. On est donc ici dans une perspective bien différente de celle de la classe habituelle ou du laboratoire des langues où l'étudiant fait des exercices de correction phonétique ou des activités orales mécaniques sans possibilité d'échange réel.

C'est dans le terrain des pratiques de correction de la prononciation que le projet León - Grenoble a accompli des expérimentations innovantes et pionnières dans ces contextes éducatifs. Les tuteurs de l'université de Grenoble ont dû affronter le travail difficile de la correction de la prononciation à distance et avec les nouvelles technologies, et ils ont bien répondu à ces expériences inédites, car ils ont inventé et appliqué trois types de techniques de correction de la prononciation : 1. Commentaires écrits sur les erreurs ou difficultés observées dans les tâches orales. 2. Réenregistrement par le natif français de l'audio envoyé par l'espagnol. 3. Insertion orale de la correction dans l'enregistrement originel de l'étudiant espagnol.

Les corrections collaboratives audiovisuelles entre étudiants constituent une autre expérience pionnière dans la recherche en didactique des langues. Nous avons essayé d'appliquer quelques présupposés théoriques des pédagogies actives, socioconstructivistes et axées sur les tâches, ainsi que des paradigmes éducatifs comme « apprendre à faire » ou «apprendre à partager». Afin de favoriser une pratique de communication authentique nous avons eu recours à la mise en situation qui suppose un scénario de correction de la prononciation (simulation des rôles d'enseignant et d'étudiant). Et nous avons enregistré des séquences vidéo en direct avec UStream.TV destinées aux tuteurs de Grenoble pour qu'ils assistent à des moments réels dans la réalisation des tâches en salle d'informatique à l'Université de León.

Finalement nous voulons rappeler que cette recherche participe d'un esprit ouvert dans le but de partager les expériences et les connaissances pour la construction d'une « intelligence collective» grâce aux nouvelles technologies. La plupart des ressources, 
outils et documents audiovisuels cités dans cet article sont disponibles en ligne dans différents espaces web : site du projet León - Grenoble, canal Campus du site UStream.TV ou du projet Flenet de l'Université de León. De cette manière nous espérons ouvrir de nouvelles voies dans l'application des nouvelles technologies pour l'enseignement des langues et en même temps laisser à la communauté scientifique des échantillons (productions orales) importants pour d'autres recherches et pratiques.

\section{BIBLIOGRAFÍA}

Blandin, B et al. (2002) Le B.A. BA de la FOAD. Paris: Forum Français de la Formation à Distance (FFFOD), juin 2002. Consulté en mars 2007 : http://www.fffod.org/

Cadree européen commun de référence pour les langues : Apprendre, Enseigner, Évaluer (CECRL). Conseil de 1' Europe, Strasbourg, 2001. Version française : http://culture2.coe.int/portfolio/documents/cadrecommun.pdf Versión española : http://cvc.cervantes.es/obref/marco/cvc_mer.pdf . Synthèse du CECRL rédigée par F. Goullier et Y. Chevillard. Académie de Toulouse. Consulté en novembre 2007: http://pedagogie.ac-toulouse.fr/anglais/cadeurop.html

CALICO, EUROCALL, IALLT (1999) Scholarly activities in computer-assisted language learning: development, pedagogical innovations and research. Joint policy statement, 1999. Consulté en novembre 2007: http://www.iallt.org/iallt_documents/ScholActCALL.pdf

Charlier, B., Deschryver, N. et Peraya, D. (2005) Apprendre en présence et à distance A la recherche des effets des dispositifs hybrides. Réseau Education Formation (REF), 15-16/09/05, Montpellier. Consulsté en janvier 2007: http://pedagogie.ac-montpellier.fr/Disciplines/maths/REF_2005/REF-

Charlier.pdf

Delors, J.et al.(1999) Les quatre piliers de l'éducation. L'education: un trésor est caché dedans. Rapport à l'UNESCO, commission internationale sur l'éducation pour le vingt et unième siècle, Editions UNESCO, 2ème edition, 1999, Paris. Synthèse web: Les quatre piliers de l'éducation: http://www.unesco.org/delors/tffrench/piliers.htm

De Rosnay, J et C. Revelli (2006) La révolte du pronétariat: Des mass média aux média des masses. Fayard Web du livre: http://www.pronetariat.com/

Develotte, C. et Mangenot, F. (2007) Discontinuités didactiques et langagières au sein d'un dispositif pédagogique en ligne ; Glottopol, n¹0, pp. 127-144. Consulté en octobre 2007: http://www.univrouen.fr/dyalang/glottopol/telecharger/numero_10/gpl10_09develotte.pdf

Develotte C., Guichon N., Kern R. (2007) "Allo Berkeley? Ici Lyon... Vous nous voyez bien?" Etude d'un dispositif d'enseignement-apprentissage en ligne synchrone franco-américain. Actes du colloque Echanger pour apprendre en ligne (EPAL). Grenoble, 7-9 juin 2007. Consulté en décembre 2007: http://w3.ugrenoble3.fr/epal/pdf/develotte-kern-guichon.pdf

Downes, S. (2005) E-Learning 2.0. ELearn Magazine (C-Publications in Trade Journals (invited article)) Association for Computing Machinery October 16, 2005. Consulté en novembre 2007: http://www.downes.ca/post/31741

Edelenbos, P., Johnstone, R., Kubanek, A (2006) Les grands principes pédagogiques sur lesquels se fonde l'enseignement des langues aux très jeunes apprenants. 
Commission européenne, octobre 2006. Consulté en novembre 2007: http://ec.europa.eu/education/policies/lang/doc/young_fr.pdf

Furstenberg, G., Levet, S., English, K., Maillet, M. (2001) Giving a Virtual Voice to the Silent Language of Culture: The Cultura Project Classroom Examples; Online article published in Language Learning \& Technology, Vol. 5, No. 1, January 2001, pp. 55-102 http://llt.msu.edu/vol5num1/furstenberg/default.html

Henri, F. et Lundgren-Cayrol, K. (1997). Apprentissage collaboratif à distance, téléconférence et télédiscussion. Montréal : LICEF. Consulté en octobre 2006: http://www.licef.teluq.uquebec.ca/Bac/fiches/f48.htm

Johnson, B. (1992) Institutional Learning, in B.-A.- Lundvall (Editor) National Systems of Innovation, Pinter, London, pp. 23-44.

Lamy, M.-N. (2006) Conversations multimodales: l'enseignement-apprentissage de l'oral à l'heure des écrans partagés. Le Français dans le monde, Recherches et applications, Les échanges en ligne dans l'apprentissage et la formation. $\mathrm{N}^{\mathrm{o}}$ 40; pp.129-138.

Lamy, M.-N. (2004) Pratique de l'oral en petits groupes via Internet: quels défis pédagogiques? in "Plurilinguisme et multimédia" - Journées d'étude - ENS LSH Lyon. Develotte, C. (organis.) Formations en langues et Internet: quels aspects collaboratifs? Jeudi 11 mars 2004: Consulté en septembre 2007: http://w1.ens-lsh.fr/labo/plumme/11042004/lamy/lamy.htm

Levy, P.(1997) L' intelligence collective. Pour une anthropologies du cyberspace, La Découverte. Paris.

Lundvall, B.A. (2002). The University in the Learning Economy. DRUID, 2. Consulté en septembre 2007: http://www.druid.dk/wp/pdf files/02-06.pdf

Marcelli A., Gaveau, D., Tokiwa, R. (2005). Utilisation de la visioconférence dans un programme de FLE : tâches communicatives et interactions orales. Alsic. Vol. 8. pp. 185-203. Consulté en novembre 2007 : http://alsic.ustrasbg.fr/v08/marcelli/alsic_v08_18-pra2.pdf

Mangenot, F. (2003) Tâches et cooperation dans deux dispositifs universitaires de formation a distance. Alsic, Université de Franche-Comté, Besançon, Vol. 6, Numéro 1, Juin 2003, pp 109-125. Consulté en juin 2005: http://alsic.ustrasbg.fr/Num10/mangenot/alsic_n10-rec10.htm

Mangenot, F. (2005) Apprendre, enseigner et former avec Internet, Conférence CUEF - Université Stendhal (11 juillet 2005). Consulté en janvier 2006: http://flenet.rediris.es/tourdetoile/MangenotCuef2005a.htm

Mangenot, F. \& Zourou, K. (2007) Pratiques tutorales correctives via Internet : le cas du français en première ligne, ALSIC, Vol. 10, Numéro spécial Tidilem. Consulté en juin 2007: http://alsic.u-strasbg.fr/v10/mangenot/alsic_v10_07rec5.htm

MECdecretos - Síntesis (RedIRIS) - Reales Decretos de enseñanzas mínimas de la Educación Primaria y de la Educación Secundaria Obligatoria (2006). Consulté en novembre 2007:

http://cvu.rediris.es/pub/bscw.cgi/d754687-4/*/*/*/*/MECdecretos_sintesis.html

O'Dowd, R. (2006) The Use of Videoconferencing and E-mail as Mediators of Intercultural Student Ethnography. In J. A. Belz \& S. L. Thorne (dir.) Computer-mediated Intercultural Foreign Language Education, Boston : Heinle \& Heinle. pp. 86-120.

Oliver, D. \& Herrington, J. (2001) Teaching and Learning Online. Perth : Edith Cowan University. 
Reffay C. et Betbeder M.-L. (2006) Spécificités des plateformes audio-graphiques synchrones dans un dispositif de formation. Le Français dans le monde, Recherches et applications, Les échanges en ligne dans l'apprentissage et la formation. $\mathrm{n}^{\circ}$ 40. pp. 124-128 Consulté en novembre 2007: http://edutice.archives-ouvertes.fr/edutice-00088404

Tomé, M. (1994) Fonética francesa para uso de estudiantes españoles, Universidad de Leon, Servicio de publicaciones, León, 84 pages.

Tomé, M. (1995), L' enseignement de la prononciation du français pour des débutants espagnols; Actas del II Coloquio Internacional de Lingüistica francesa, Universidad de Sevilla, Sevilla, 2-4 noviembre, 1995, pp. 31-35. Consulté en novembre

2007: http://www3.unileon.es/dp/dfm/flenet/phoneticours1.html\#Tome95

Tomé, M. (2004) Étude sur le dispositif "Campus virtuel FLE - Universidad de León" et ses applications pédagogiques. I Congrès International TIC et Autonomie Appliquées à l'Apprentissage des langues (TAAAL), Université Jaume I, Castellón de la Plana. Consulté en janvier 2006: http://www3.unileon.es/dp/dfm/flenet/activi5.html\#EDC

Tomé, M. (2006) L'enseignant de FLE et les ressources Internet, Çédille. Revista de estudios franceses, $\mathrm{n}^{\mathrm{o}} 2$ (2006), pp. 114-133. Consulté en juin 2006: http://webpages.ull.es/users/cedille/dos/tome.pdf

Tomé, M. (2007) Applications pédagogiques des weblogs dans le Campus Virtuel FLE, Estudios Humanísticos - Filología, $\mathrm{N}^{\mathrm{o}}$ 29, 341-373. Universiad de Léon. Version électronique Revue Tourdetoile, $\mathrm{n}^{\circ}$ 10. Consulté en juin 2007: http://flenet.rediris.es/tourdetoile/Tome_BlogsCampusEH07.html

Tomé, M. (2007) Expériences pédagogiques dans le Campus Virtuel FLE; ELA : Etudes de Linguistique Appliquée: Revue de didactologie des langues-cultures et de lexiculturologie; D'autres espaces pour les cultures, no 146, 2007, pp.179188. Paris. Klincksieck.

Vetter A. (2004) Les spécificités du tutorat à distance à l'Open University : enseigner les langues avec Lyceum; Alsic, vol. 7, 1, novembre 2004, pp. 107-129. Consulté en novembre 2007: http://alsic.u-strasbg.fr/v07/vetter/alsic_v07_06pra2.htm

Zourou, K. (2005) Apprentissages collectifs médiatisés et didactique des langues : instrumentation, dispositifs et accompagnement pédagogique. Thèse de doctorat en sciences du langage soutenue à l'université Stendhal - Grenoble 3. Consulté en septembre 2007: http://tel.archives-ouvertes.fr/tel-00119459

Cultura (1997-2008) créé par G. Furstenberg, S. Waryn et S. Levet du Foreign Languages and Literatures in Massachusetts Institute of Technology (MIT). Partenaires: MIT (Cambridge, USA), Supaéro (Toulouse, France), INT (Evry, France), Lenox High (USA) \& Lycée Marcellin Berthelot (France), Brown University (Providence, USA) Paris II (Paris, France), Ecole Polytechnique (Paris, France). Adresse internet: http://web.mit.edu/french/culturaNEH/

Le français en (première) ligne (2002-2008). Création et tutorat d'activités multimédias pour des apprenants distants. Créé par C.Develotte et F.Mangenot. Partenaires: Université de Sydney, Ecole normale supérieure Lettres et sciences humaines (ENS LSH) de Lyon, Université de Franche-Comté, Université Monash de Melbourne, Université Stendhal - Grenoble 3, Université de Léon (Espagne), Northern Virginia Community College (Etats-Unis), Université Lyon 2, Université Sophia de Tokyo (Japon) et the University of California, 
Berkeley (Etats-Unis). Adresse internet: http://w3.u-grenoble3.fr/fle-1ligne/index.htm

Projet León - Grenoble (2005-2008) Partenaires: Université Stendhal - Grenoble 3 (France), F. Mangenot, laboratoire Lidilem et Universidad de León (España), M. Tomé, Campus Virtuel FLE. Adresse internet : http://flenet.rediris.es/projetLG/webProjetLG05.htm. Plateforme Moodle Dpto. Filología Moderna, Universidad de León : http://www3.unileon.es/dp/dfm/moodle/ 